Punjab University Journal of Mathematics (2021),53(11),779-791

https://doi.org/10.52280/pujm.2021.531102

\title{
Families of Means-Based Modified Newtons Method for Solving Nonlinear Models
}

\author{
Oghovese Ogbereyivwe $^{1}$ and Veronica Ojo-Orobosa ${ }^{2}$ \\ Department of Mathematics and Statistics, \\ Delta State University of Science and Technology, Ozoro, \\ Delta State, Nigeria \\ Email: ${ }^{1}$ oghoveseogbereyivwe@dspz.edu.ng,ogho2015@gmail.com \\ Email: ${ }^{2}$ orobosavera@gmail.com \\ ORCID ID: ${ }^{1}$ 0000-0001-9810-2299
}

Received: 29 September, 2021 / Accepted: 04 November, 2021 / Published online:25 November, 2021

\begin{abstract}
In literature, the arithmetic mean of the two functions in the denominator of the second step of order three Weerakoon and Fernando (2000) iterative method have been replaced with other different means. However, these actions have not improve its order of convergence. To improve the order of convergence of these modified methods, a generic family of iterative methods that involve two weight functions and a generic consequential function for replacement of means is proposed. The analysis of convergence carried out on the families of methods, shows that they are of fourth order convergence and requires evaluation of three functions per iteration cycle. Further, the flexibility of the weight functions enables the re-discovery of some existing and construction of new families of iterative methods. Some concrete members of the family of methods are applied to solve some nonlinear equations and real life problems that are modeled into nonlinear equations.
\end{abstract}

\section{AMS (MOS) Subject Classification Codes:65H05; 65D05}

Key Words: Nonlinear Model; Mean-Based; Weight Functions; Iterative Methods

\section{INTRODUCTION}

The area of numerical mathematics that involved the development of new iterative methods (IM) for determining the solution of nonlinear equations (NE) have attracted researchers' interest for decades. This is because many real life phenomena are continued to be modelled into NE and requires the application of efficient IM to determine their solutions. The generic form of Nonlinear models (NLM) is:

$$
f(x)=0,
$$


where $x \in R, f: D \subset R \rightarrow R$ is functional.

The Newton method (NM)[24] is the most popular IM for determining the solution $x^{*}$ of (1.1) and is given as:

$$
x_{k+1}=x_{k}-s_{k}, \quad k=0,1,2, \cdots
$$

where $s_{k}=\frac{f\left(x_{k}\right)}{f^{\prime}\left(x_{k}\right)}$. The convergence order (CO) of the IM (1.2) is 2 and requires the evaluation of two distinct functions per iteration cycle.

Over the years, plethora of IM for determining the solution $x^{*}$ of (1.1) have been developed in literature. These developed methods are usually variants of the method (1.2) and in most cases targeted at improving its $\mathrm{CO}$ and efficiency index $(E I)$. The $E I$ of a method with $\mathrm{CO}$ $\rho$ is determined using $\rho^{\frac{1}{q}},[18]$. In order to improve the CO and $E I$ of (1.2), many authors have used different techniques. A good review on some of these techniques such as the geometric, functional, composition, sampling, Adomain decomposition, rational function and weight function techniques can be found in $[1,15,16,17]$ and reference therein.

The Weerakoon and Fernando (W-F) method in [25] is one modified form of the NM (1.2), put forward by replacing the function $f^{\prime}(x)$ in (1.2) with the arithmetic mean of the functions $f(x)$ and $f^{\prime}(w)$. It is given as

$$
\begin{gathered}
w_{k}=x_{k}-s_{k}, \\
x_{k+1}=x_{k}-\frac{2 f\left(x_{k}\right)}{f^{\prime}\left(x_{k}\right)+f^{\prime}\left(w_{k}\right)},
\end{gathered}
$$

with CO three and $E I=1.4422$. Since the W-F method was put forward, many authors have replaced the arithmetic mean used in [25] with other types of means to developed many IM of CO three. For instance, Harmonic mean was used in [19], geometric mean was employed in [14], Heronian mean and Quadratic mean were utilized in [23] and [26] respectively. Further, in $[9,10]$ the Stolarsky, Gini, Power, $p$-Logarithm, Heron, Contraharmonic and Symmetric means where used to develop families of means-based CO three methods which contain methods from papers [25,19,14,23] and others.

To improve the $\mathrm{CO}$ and $E I$ of the third orders means-based methods, many authors have introduced to the second step of the methods, a unique weight function that contain one additional new function evaluation which ensures the method attain higher $C O$ and $E I$, see $[13,4,11,3]$. In quest for contribution to these research trends, we introduce two weight functions to the second step of means-based iterative function which led to the development of families of modified means-based modified NM for approximating the solution of (1.1). The rest part of this paper is arranged in the following format. Section 2, presents the developmental stages of the new families of IM and their convergence analysis. While the numerical experience of the developed and compared methods on theoretical problems are presented in Section 3, the application to real life problems are presented in Section 4. The last section of this document, contains the conclusion and suggested further research areas.

\section{Method Formulation}

Consider the modified NM given as: 


$$
x_{k+1}=x_{k}-\frac{f\left(x_{k}\right)}{M_{T}\left[f^{\prime}\left(x_{k}\right), f^{\prime}\left(w_{k}\right)\right]}, k=0,1,2 . \cdots
$$

where $w_{k}$ is Newton method iterative step and $M_{T}$ is any type of mean of the functions derivatives $f^{\prime}\left(x_{k}\right)$ and $f^{\prime}\left(w_{k}\right)$.

Define a transformation $\Omega$ on the second step of the method in (2.4) such that

$$
\Omega\left(\frac{f\left(x_{k}\right)}{M_{T}\left[f^{\prime}\left(x_{k}\right), f^{\prime}\left(w_{k}\right)\right]}\right)=\Phi_{M_{T}}\left(s_{k}, v_{k}\right),
$$

where $s=\frac{f\left(x_{k}\right)}{f^{\prime}\left(x_{k}\right)}, v=\frac{f^{\prime}\left(w_{k}\right)}{f^{\prime}\left(x_{k}\right)}$ and $\Phi_{M_{T}}$ is a real valued bi-variate function of $s$ and $v$ which is the result of the transformation $\Omega$ when a type of mean is used in $M_{T}$. For this reason, $\Phi_{M_{T}}$ is referred to as the consequential transformation function when any type of mean is used as $M_{T}$. Consequently, (2.4) becomes

$$
x_{k+1}=x_{k}-\Phi_{M_{T}}\left(s_{k}, v_{k}\right), k=0,1,2, \cdots
$$

Suppose $M_{T}$ is Arithmetic mean, that is $M_{T}=\frac{f^{\prime}\left(x_{k}\right)+f^{\prime}\left(w_{k}\right)}{2}$ and after subjecting it to the transformation in (2.5), the consequential IM in (2.4) becomes

$$
x_{k+1}=x_{k}-\frac{2 s_{k}}{1+v_{k}}, k=0,1,2 . \cdots
$$

The IM in (2.7) is the W-F method [25] with its corrector function expressed as a function of $s_{k}$ and $w_{k}$.

Other similar modified form of method (2.4) that are of CO three have been obtained by using different type of mean in $M_{T}$ and then subjecting it to the transformation in (2.5). For example:

For Harmonic mean [19], $M_{H}=2\left(\frac{1}{f^{\prime}\left(x_{k}\right)}+\frac{1}{f^{\prime}\left(w_{k}\right)}\right)^{-1}$, we get

$$
\Phi_{M_{H}}\left(s_{k}, v_{k}\right)=\frac{s_{k}}{2}\left(1+\frac{1}{v_{k}}\right), k=0,1,2 . \cdots
$$

For Geometric mean [14], $M_{G}=\sqrt{f^{\prime}\left(x_{k}\right) f^{\prime}\left(w_{k}\right)}$, we get

$$
\Phi_{M_{G}}\left(s_{k}, v_{k}\right)=\frac{s_{k}}{\sqrt{v_{k}}}, k=0,1,2 . \cdots
$$

For Hearonian mean [23], $M_{H e}=\frac{2}{3} \frac{f^{\prime}\left(x_{k}\right)+f^{\prime}\left(w_{k}\right)}{2}+\frac{1}{3} \sqrt{f^{\prime}\left(x_{k}\right) f^{\prime}\left(w_{k}\right)}$, yield

$$
\Phi_{M_{H e}}\left(s_{k}, v_{k}\right)=\frac{3 s_{k}}{1+v_{k}+\sqrt{v_{k}}}, k=0,1,2 . \cdots
$$

For Quadratic mean [26], $M_{Q}=\sqrt{\frac{1}{2}\left(f^{\prime 2}\left(x_{k}\right)+f^{\prime 2}\left(w_{k}\right)\right)}$, yield

$$
\Phi_{M_{Q}}\left(s_{k}, v_{k}\right)=\frac{s_{k}}{\sqrt{\frac{1}{2}+\frac{1}{2} v_{k}^{2}}}, k=0,1,2 . \cdots
$$


2.1. The families of the means-based methods. In order to make the method (2.7) and all other methods developed from (2.4) by using the transformation of the types of mean given in Equations (2.8 - 2.11) attain CO four, the following family of IM is suggested.

$$
\begin{gathered}
y_{k}=x_{k}-\alpha s_{k} ; \\
x_{k+1}=x_{k}-\Phi_{M_{T}}\left(s_{k}, v_{k}\right) H\left(s_{k}\right) G\left(v_{k}\right), \quad k=0,1,2, \cdots
\end{gathered}
$$

where $\alpha \subset R \backslash\{0\}$ a free parameter, $H\left(s_{k}\right)$ and $G\left(v_{k}\right)$ are real-valued weight functions that ensures the $\mathrm{CO}$ of (2.12) attain four by utilizing fixed number of functions evaluations per iteration cycle. If method (2.12) is established to converge with order four, then for any concrete method of it will require the evaluation of three functions per iteration cycle and consequently, its $E I$ will be 1.5873 .

Next we establish the convergence of the families of means-based IM given in (2.12) for different types of mean used in $M_{T}$. The Taylor's expansion technique also used in literature $[15,16,17,13]$ and some reference therein, was used in proving the convergence of the method. First, the Proposition 2.1 is considered.

Proposition 2.1. Let $f: D \subset R \rightarrow R$ be a real valued function that is at least threetimes differentiable and that $f^{\prime}(\cdot) \neq 0$ in $D$. Then for every type of mean used in $M_{T}$, the function $\Phi_{M_{T}}\left(s_{k}, v_{k}\right)$ in (2.12) contributes an error to the iterative process given as:

$$
\begin{aligned}
e_{\Phi_{M_{T_{k}}}=e_{k}+(\alpha-1) c_{2} e_{k}^{2}} & \left(B_{M_{T}} c_{2}^{2}+D_{M_{T}} c_{3}\right) e_{k}^{3} \\
+ & \left(E_{M_{T}} c_{2}^{3}+F_{M_{T}} c_{2} c_{3}+J_{M_{T}} c_{4}\right) e_{k}^{4}+O\left(e_{k}^{5}\right)
\end{aligned}
$$

where $B_{M_{T}}, D_{M_{T}}, E_{M_{T}}, F_{M_{T}}$ and $J_{M_{T}}$ are constants expressed in terms of $\alpha$.

Theorem 2.1. Assume that $f: D \subset R \rightarrow R$ is a real valued function that is at least threetimes differentiable such that $f^{\prime}(\cdot) \neq 0$ in $D$. If $x_{0}$ is an initial guess close to $x^{*} \subset D$, then the sequence of approximations $\left\{x_{k}\right\}_{k \geq 0},\left(x_{k} \in D\right)$ generated using the families of methods (2.12) for any mean type $M_{T}$ used, converges to $x^{*}$ with order four and generic error equation

$$
\begin{aligned}
e_{k+1}= & \frac{1}{162 G(1)}\left(54 c_{2} c_{3}\left(-2+12 B_{M_{T}}-3 F_{M_{T}}\right) G(1)-2 c_{2}^{3}\left(\left(-327+540 B_{M_{T}}\right.\right.\right. \\
& \left.\left.\left.+81 E_{M_{T}}\right) G(1)-32 G^{\prime \prime}(1)\right)-3 G(1)\left(9 G(1) H^{\prime \prime \prime}(0)+c_{4}\left(52+54 J_{M_{T}}\right)\right)\right) e_{k}^{4} \\
& +O\left(e_{k}^{5}\right)
\end{aligned}
$$

where $c_{n}=\frac{1}{n !} \frac{f^{n}\left(x^{*}\right)}{f^{\prime}\left(x^{*}\right)}, n \geq 2$, provided the weight functions $H\left(s_{k}\right)$ and $G\left(v_{k}\right)$ satisfy the following conditions:

$\alpha=\frac{2}{3}, \quad D_{M_{T}}=-\alpha, \quad H(0)=\frac{1}{G(1)}, \quad G(1) \neq 0, \quad H^{\prime}(0)=H^{\prime \prime}(0), \quad H^{\prime \prime \prime}(0)<\infty$, $G^{\prime}(1)=\frac{1}{2} G(1), \quad G^{\prime \prime}(1)=\left(\frac{10-9 B_{M_{T}}}{8}\right) G(1), \quad G^{\prime \prime \prime}(1)<\infty$.

Proof. Let the kth iteration error be $e_{k+1}=x_{k}-x^{*}$. If $x=x_{k}$ in the Taylor series expansion of $f(x)$ and $f^{\prime}(x)$ about $x^{*}$, then 


$$
f\left(x_{k}\right)=f^{\prime}\left(x^{*}\right)\left[e_{k}+\sum_{n=2}^{4} c_{n} e_{k}^{n}+O\left(e_{k}^{5}\right)\right], k=0,1,2, \cdots
$$

and

$$
f^{\prime}\left(x_{k}\right)=f^{\prime}\left(x^{*}\right)\left[1+\sum_{n=2}^{4} c_{n} e_{k}^{n-1}+O\left(e_{k}^{5}\right)\right], k=0,1,2, \cdots
$$

where $c_{n}=\frac{1}{n !} \frac{f^{n}\left(x^{*}\right)}{f^{\prime}\left(x^{*}\right)}, n \geq 2$.

Using (2.15) and (2.16), the following expressions are obtained.

$$
\begin{aligned}
s_{k}=\frac{f\left(x_{k}\right)}{f^{\prime}\left(x_{k}\right)}= & e_{k}-c_{2} e_{k}^{2}+\left(2 c_{2}^{2}-2 c_{3}\right) e_{k}^{3}+\left(-4 c_{2}^{3}+7 c_{2} c_{3}-c_{4}\right) e_{k}^{4} \\
& +\left(8 c_{2}^{4}-20 c_{2}^{2} c_{3}+6 c_{3}^{2}+10 c_{2} c_{4}-4 c_{5}\right) e_{k}^{5}+O\left(e_{k}^{6}\right)
\end{aligned}
$$

and

$$
\begin{aligned}
w_{k}= & x_{k}-\alpha \frac{f\left(x_{k}\right)}{f^{\prime}\left(x_{k}\right)} \\
= & x^{*}+(1-\alpha) e_{k}+\alpha c_{2} e_{k}^{2}+\alpha\left(-2 c_{2}^{2}+2 c_{3}\right) e_{k}^{3}+\alpha\left(4 c_{2}^{3}-7 c_{2} c_{3}+3 c_{4}\right) e_{k}^{4} \\
& +O\left(e_{k}^{5}\right)
\end{aligned}
$$

Now by expanding $f^{\prime}\left(w_{k}\right)$ using Taylor series expansion, we have:

$$
\begin{aligned}
f^{\prime}\left(w_{k}\right) & =f^{\prime}\left(x^{*}\right)\left[1+2(1-\alpha) c_{2} e_{k}+\left(2 \alpha c_{2}^{2}+3(1-\alpha)^{2} c_{3}\right) e_{k}^{2}+\left(6(1-\alpha) \alpha c_{2} c_{3}\right.\right. \\
& \left.+2 \alpha c_{2}\left(-2 c_{2}^{2}+2 c_{3}\right)+4(1-\alpha)^{3} c_{4}\right) e_{k}^{3}+\left(3 c_{3}\left(\alpha \alpha^{2} c_{2}^{2}+2(1-\alpha) \alpha\left(-2 c_{2}^{2}+2 c_{3}\right)\right)\right. \\
& \left.\left.+12(1-\alpha)^{2} \alpha c_{2} c_{4}+2 \alpha c_{2}\left(4 c_{2}^{3}-7 c_{2} c_{3}+3 c_{4}\right)+5(1-\alpha)^{4} c_{5}\right) e_{k}^{4}+O\left(e_{k}^{5}\right)\right]
\end{aligned}
$$

The variable $v_{k}$ of the weight function $G\left(v_{k}\right)$ can be express as

$$
\begin{aligned}
v_{k} & =\frac{f^{\prime}\left(w_{k}\right)}{f^{\prime}\left(x_{k}\right)} \\
& =1-2 \alpha c_{2} e_{k}+3 \alpha\left(2 c_{2}^{2}+(-2+\alpha) c_{3}\right) e_{k}^{2}-4 \alpha\left(4 c_{2}^{3}+(-7+3 \alpha)\right. \\
& \left.+\left(3-3 \alpha+\alpha^{2}\right) c_{4}\right) e_{k}^{3}+\alpha\left(40 c_{2}^{4}+(-100+39 \alpha) c_{2}^{2} c_{3}+(30-21 \alpha) c_{3}^{2}\right. \\
& \left.+2\left(25-24 \alpha+10 \alpha^{2}\right) c_{2} c_{4}+5\left(-4+6 \alpha-4 \alpha^{2}+\alpha^{3}\right) c_{5}\right) c_{k}^{4}+O\left(c_{k}^{5}\right)
\end{aligned}
$$

and from Proposition 2.1,

$$
\begin{aligned}
\Phi_{M_{T}}\left(s_{k}, v_{k}\right)=e_{k}+(\alpha-1) c_{2} e_{k}^{2} & +\left(B_{M_{T}} c_{2}^{2}+D_{M_{T}} c_{3}\right) e_{k}^{3} \\
+ & \left(E_{M_{T}} c_{2}^{3}+F_{M_{T}} c_{2} c_{3}+J_{M_{T}} c_{4}\right) e_{k}^{4}+O\left(e_{k}^{5}\right)
\end{aligned}
$$

The Taylor expansion of the weight functions $H\left(s_{k}\right)$ and $G\left(v_{k}\right)$ about $s_{k}=0$ and $v_{k}=1$ respectively, are 


$$
H\left(s_{k}\right)=H(0)+s H^{\prime}(0)+\frac{1}{2} s^{2} H^{\prime \prime}(0)+\frac{1}{6} s^{3} H^{\prime \prime \prime}(0)+\cdots
$$

and

$$
G\left(v_{k}\right)=G(1)+(v-1) G^{\prime}(1)+\frac{1}{2}(v-1)^{2} G^{\prime \prime}(1)+\frac{1}{6}(v-1)^{3} G^{\prime \prime \prime}(1)+\cdots
$$

Now using (2.21), (2.22), and (2.23) in the second step of (2.12), we get

$$
\begin{aligned}
x_{k+1}= & (1-G(1) H(0)) e_{k}+\left(c_{2}\left(G(1)-\alpha G(1)+2 \alpha G^{\prime}(1)\right) H(1)-G(1) H^{\prime}(0)\right) e_{k}^{2} \\
& +\left(-\left(\left(B_{M_{T}} c_{2}^{2}+c_{3} D_{M_{T}}\right) G(1) H(0)\right)-\alpha\left(3(\alpha-2) c_{3} G^{\prime}(1)\right)+2 c_{3}^{2}\left(3 G^{\prime}(1)\right.\right. \\
& \left.+\alpha G^{\prime \prime}(1)\right) H(0)+c_{2} G(1) H^{\prime}(0)+2 \alpha c_{2} G^{\prime}(1) H^{\prime}(0) \\
& \left.+(\alpha+1) c_{2}\left(2 \alpha c_{2} G^{\prime}(1) H(0)-G(0) H^{\prime}(0)\right)-\frac{G(1) H^{\prime}(0)}{2}\right) e_{k}^{3} \\
& +\left(4 \alpha^{3} c_{4} G^{\prime}(1) H(0)-\frac{1}{3}\left(c _ { 2 } ^ { 3 } \left(3 E_{M_{T}} G(0)+2 \alpha((9 \alpha\right.\right.\right. \\
& \left.\left.\left.-3\left(11+B_{M_{T}}\right)\right) G^{\prime}(1)+\alpha\left(3(\alpha-7) G^{\prime \prime}(1)-2 \alpha G^{\prime \prime \prime}(0)\right)\right)\right) H(0) \\
& +c_{2}^{2}\left((-3+\alpha-B) G(1)+2 \alpha\left((\alpha-5) G^{\prime}(1)-\alpha G^{\prime \prime}(1)\right)\right) H^{\prime}(0) \\
& +6 \alpha G^{\prime}(1)\left(2 c_{4} H(0)+c_{3} H^{\prime}(0)\right)-3 \alpha^{2} G^{\prime}(1)\left(4 c_{4} H(0)+c_{3} H^{\prime}(0)\right) \cdots \\
& \left.-\frac{1}{6} G(1)\left(6 c_{3}\left(D_{M_{T}}-2\right) H^{\prime}(0)+H^{\prime \prime \prime}(0)+6 c_{4} H(0) H(0) J_{M_{T}}\right)\right) e_{k}^{4} \\
& +O\left(e_{k}^{5}\right)
\end{aligned}
$$

The method (2.12) converges to $x^{*}$ if the first, second, and third term of (2.24) vanish. This is achieved when the following set of system of equations holds.

$$
\begin{aligned}
& G(1) H^{\prime}(0)=0 \\
& 1-G(1) H(0)=0 \\
& \left(G(1)-\alpha G(1)+2 \alpha G^{\prime}(1)\right) H(0)=0 \\
& \frac{1}{2} G(1) H^{\prime}(0)=0 \\
& 2 G^{\prime}(1)(2 \alpha-1)-B_{M_{T}} G(1)=0 \\
& 3 \alpha G^{\prime}(1)(2-\alpha)-D_{M_{T}} G(1)=0 \\
& 2 \alpha\left(3 G^{\prime}(1)-\alpha G^{\prime \prime}(1)\right)=0
\end{aligned}
$$

The system of equations (2.25) is satisfied if

$$
\begin{aligned}
& \alpha=\frac{2}{3}, \quad D_{M_{T}}=-\alpha, H(0)=\frac{1}{G(1)}, \quad G(1) \neq 0, H^{\prime}(0)=H^{\prime \prime}(0)=0, \\
& H^{\prime \prime \prime}(0) \leq \infty, \quad G^{\prime}(1)=-\frac{1}{4} G(1), \\
& G^{\prime \prime}(1)=\left(\frac{10-9 B_{M_{T}}}{8}\right) G(1), G^{\prime \prime \prime}(1)<\infty .
\end{aligned}
$$


By substituting the weights conditions (2.26) in (2.22) and (2.23), the following general error equation of any method constructed from (2.12) is obtained as:

$$
\begin{aligned}
e_{k+1}= & \alpha+\frac{1}{162 G(1)}\left(54 c_{2} c_{3}\left(12 B_{M_{T}}-3 F_{M_{T}}-2\right) G(1)-2 c_{2}^{3}\left(\left(540 B_{M_{T}}+81 E_{M_{T}}\right.\right.\right. \\
& \left.\left.-327) G(0)-32 G^{\prime \prime \prime}(0)\right)-3 G(1)\left(9 G(1) H^{\prime \prime \prime}(0)+c_{4}\left(52+54 J_{M_{T}}\right)\right)\right) e_{k}^{4} \\
& +O\left(e_{k}^{5}\right)
\end{aligned}
$$

The error equation in (2.27) establishes the claim that for any concrete method derived from (2.12), will have $\mathrm{CO}$ four.

Remark 2.1: For any two weight functions $H(s)$ and $G(v)$ jointly satisfying the conditions in (2.26), a family of CO four means-based IM for solving (1.1) can be put forward. For instance:

$H(s)=\left(\frac{1}{\delta}+\theta s^{3}\right)$ and $G(v)=\delta-\frac{1}{4} \delta(v-1)+\frac{\eta}{2} \delta(v-1)^{2}+\tau(v-1)^{3}$

where $\delta \neq 0, \theta<\infty$ and $\tau<\infty$ are free constant. These weight functions when substituted in (2.12) yield a family of methods given as:

$$
\begin{aligned}
y_{k} & =x_{k}-\frac{2}{3} s_{k} \\
x_{k+1} & =x_{k}-\Phi_{M_{T}}\left(s_{k}, v_{k}\right)\left(\frac{1}{\delta}+\theta s_{k}^{3}\right)\left(\delta-\frac{1}{4} \delta \sigma+\frac{\eta}{2} \delta \sigma^{2}+\tau \sigma^{3}\right) \\
k & =0,1,2, \cdots
\end{aligned}
$$

where $\sigma=v_{k}-1$ and $\eta=\left(\frac{10-9 B_{M_{T}}}{8}\right)$.

We state here that the family of order four methods developed in $[13,14]$ is a consequential member of the method in (2.28) with $\theta=\tau=0$ ( More details on this can be seen in Remark 2.3). For this reason method (2.28) is referred to as family of families of CO four methods for solving (1.1).

Remark 2.2: It is important to note that the variable $\eta$ (depending on $B_{M_{T}}$ ) is responsible for the differences in IM developed from (2.28) using different types of means in $M_{T}$. Using Proposition 2.2, the values of $\eta$ when different types of means are used in $M_{T}$ are presented in Table 1. 
TABLE 1. Values of $\eta$ for different methods.

\begin{tabular}{ccc}
\hline$M_{T}$ & $B_{M_{T}}$ & $\eta$ \\
\hline Arithmetic mean & $2-4 \alpha+\alpha^{2}$ & $\frac{3}{2}$ \\
Harmonic mean & $2(\alpha-1)^{2}$ & 1 \\
Geometric mean & $\frac{1}{2}\left(\alpha^{2}-8 \alpha+4\right)$ & $\frac{5}{4}$ \\
Hearonian mean & $\frac{7}{6} \alpha^{2}-4 \alpha+2$ & $\frac{17}{12}$ \\
Quadratic mean & $\frac{1}{2}\left(2-4 \alpha+\alpha^{2}\right)$ & $\frac{7}{4}$ \\
\hline
\end{tabular}

Remark 2.3: To construct concrete form of the method in (2.28) for a chosen $M_{T}$ (corresponding to $\eta$ ), arbitrary values are assigned to the free parameters $\delta, \theta$ and $\tau$. For instance, if $\eta=\frac{3}{2}, \delta=1, \theta=$ $\tau=0$ in (2.28), then we have the method put forward in Lofti (2014)[13] and Chicharro et al.,(2019) [4] denoted as $M_{1}$ and given as:

$$
x_{k+1}=x_{k}-\frac{2 s_{k}}{1+v_{k}}\left(2-\frac{7}{4} v_{k}+\frac{3}{4} v_{k}^{2}\right) .
$$

Some other typical methods are presented in Table 2.

TABLE 2. Concrete forms of the family of methods and their error equations

\begin{tabular}{ccccccc}
\hline Methods & $\eta$ & $\delta$ & $\theta$ & $\tau$ & $e_{k+1}$ & Remark \\
\hline$M_{1}$ & $3 / 2$ & 1 & 0 & 0 & $\frac{1}{9}\left(33 c_{2}^{3}-c_{2} c_{3}+c_{4}\right) e_{k}^{4}$ & Method in [13,4] \\
$M_{2}$ & $3 / 2$ & 1 & 1 & 1 & $\left(\frac{1}{9} c_{4}-c_{2} c_{3}+\frac{329}{81} c_{2}^{3}-\frac{1}{6}\right) e_{k}^{4}$ & New Method \\
$M_{3}$ & 1 & 1 & 0 & 0 & $\left(\frac{75}{27} c_{2}^{3}-c_{2} c_{3}+\frac{c_{4}}{9}\right) e_{k}^{4}$ & Method in [4] \\
$M_{4}$ & 1 & 2 & 2 & 6 & $\frac{1}{9}\left(37 c_{2}^{3}-9 c_{2} c_{3}+c_{4}-6\right) e_{k}^{4}$ & New Method \\
$M_{5}$ & $\frac{5}{4}$ & 1 & 0 & 0 & $\left(\frac{89}{27} c_{2}^{3}-c_{2} c_{3}+\frac{c_{4}}{9}\right) e_{k}^{4}$ & Method in [4] \\
$M_{6}$ & $\frac{5}{4}$ & $\frac{1}{2}$ & 3 & 0 & $\left(\frac{89}{27} c_{2}^{3}-c_{2} c_{3}+\frac{c_{4}}{9}-\frac{1}{4}\right) e_{k}^{4}$ & New Method \\
$M_{7}$ & $\frac{17}{12}$ & 1 & 0 & 0 & $\left(\frac{287}{81} c_{2}^{3}-c_{2} c_{3}+\frac{c_{4}}{9}\right) e_{k}^{4}$ & Method in [4] \\
$M_{8}$ & $\frac{17}{12}$ & 3 & 1 & 1 & $\left(\frac{883}{243} c_{2}^{3}-c_{2} c_{3}+\frac{c_{4}}{9}-\frac{1}{2}\right) e_{k}^{4}$ & New Method \\
$M_{9}$ & $\frac{7}{4}$ & 1 & 0 & 0 & $\left(\frac{109}{27} c_{2}^{3}-c_{2} c_{3}+\frac{c_{4}}{9}\right) e_{k}^{4}$ & Method in [4] \\
$M_{10}$ & $\frac{7}{4}$ & 1 & 1 & 1 & $\left(\frac{3599}{81} c_{2}^{3}-c_{2} c_{3}+\frac{c_{4}}{9}-\frac{1}{6}\right) e_{k}^{4}$ & New Method \\
\hline
\end{tabular}

\section{NUMERICAL IMPLEMENTATION}

To demonstrate the applicability of the developed iterative methods, some standard nonlinear problems in literature are solved. The performance of some concrete methods of the developed families of methods presented in Table 2 are compared with some existing fourth order method in Sharma et al. [21] (SM)

$$
x_{k+1}=x_{k}-\frac{4 f\left(x_{k}\right)}{f^{\prime}\left(x_{k}\right)+3 f^{\prime}\left(w_{k}\right)}\left(1+s_{k}^{3}\right)-\frac{9}{16}\left(\frac{\phi}{f^{\prime}\left(x_{k}\right)}\right)^{2} s_{k}^{3} \text {, }
$$


where $\phi=\frac{f^{\prime}\left(x_{k}\right)-f^{\prime}\left(w_{k}\right)}{s_{k}}$.

and Chun et al. [6] (CM)

$$
x_{k+1}=x_{k}-\frac{f^{\prime}\left(x_{k}\right)+3 f^{\prime}\left(w_{k}\right)}{2 f^{\prime}\left(x_{k}\right)-f^{\prime}\left(w_{k}\right)} \frac{f\left(x_{k}\right)}{f^{\prime}\left(x_{k}\right)} .
$$

All computer programs written for computations were implemented in MAPLE 2017 version environment, with 1000 digits of mantissa on Intel Celeron(R) CPU $1.6 \mathrm{GHz}$ with $2 \mathrm{~GB}$ of RAM processor. The stopping criteria used for all program is $\left|f\left(x_{k+1}\right)\right| \leq 10^{-100}$. The metrics used for comparison includes Number of Iterations (Iter), Absolute value of function of last iteration value $\left|f\left(x_{k+1}\right)\right|$ and Computational local convergence order due to Petkovic $\rho_{c o c}[8]$ given as,

$$
\rho_{c o c}=\frac{\log \left|f\left(x_{k+1}\right)\right|}{\log \left|f\left(x_{k}\right)\right|} .
$$

The developed methods $\left(M_{2 j}, j=1,2,3,4,5\right)$ and the compared methods SM and CM were used to approximate the solution of the nonlinear problems under same computation conditions and computation results obtained are presented in Table 3. The test functions $f_{1}(x)=\sin (x)-x^{2}+1$ and $f_{2}(x)=(x-1)^{3}-1$ taken from [5] were adopted for numerical test:

TABLE 3. Methods results comparison for the test functions

\begin{tabular}{cccccc}
\hline$f_{i}(x)$ & Methods & $x_{0}$ & iter & $\left|f\left(x_{k+1}\right)\right|$ & $\rho_{\text {coc }}$ \\
\hline \multirow{6}{*}{$f_{1}(x)$} & $\mathrm{SM}$ & & 5 & $2.9346 e-397$ & 4.0182 \\
& $\mathrm{CM}$ & & 4 & $1.2067 e-154$ & 4.0538 \\
& $M_{1}$ & & 4 & $4.8394 e-121$ & 4.0493 \\
& $M_{2}$ & 2 & 4 & $3.4228 e-107$ & 4.0953 \\
& $M_{4}$ & & 5 & $9.9384 e-168$ & 4.0000 \\
& $M_{6}$ & & 5 & $2.7762 e-286$ & 3.9722 \\
& $M_{8}$ & & 5 & $1.8910 e-165$ & 4.0244 \\
& $M_{10}$ & & 4 & $3.9356 e-104$ & 4.0856 \\
\hline \multirow{6}{*}{$f_{2}(x)$} & $\mathrm{SM}$ & & 4 & $1.6738 e-108$ & 4.0000 \\
& $\mathrm{CM}$ & & 4 & $1.2469 e-122$ & 3.9355 \\
& $M_{1}$ & & 5 & $2.5977 e-337$ & 4.0119 \\
& $M_{2}$ & & 5 & $8.2931 e-364$ & 4.0000 \\
& $M_{4}$ & 2.5 & 5 & $8.6029 e-381$ & 4.0105 \\
& $M_{6}$ & & 4 & $1.6872 e-204$ & 4.0000 \\
& $M_{8}$ & & 4 & $2.1119 e-101$ & 4.0400 \\
& $M_{10}$ & & 5 & $2.3967 e-351$ & 3.9889 \\
\hline
\end{tabular}

3.1. Results discussion. From Table 3, the numerical experience on some concrete forms $\left(M_{2 j}, j=1,2,3,4,5\right)$ of the developed family of families of methods (3.28) agrees with theoretical order of convergence obtained. For instance, it can be seen from the last column of Table 3 that the computational order of convergence $\rho_{c o c}$ of $\left(M_{2 j}, j=1,2,3,4,5\right)$ is four . It is also worthy of note that the developed method solved all the test problems with error margins that are competitive with methods compared. 


\section{Some Applications to Real Life Models}

In this section, some concrete forms of the developed methods $\left(M_{2 j}, j=1,2,3,4,5\right)$ are applied to solve some real life problems that have been expressed in nonlinear equations. Their numerical results were compared with the results by the methods SM and CM.

Application 1: (Colebrook-White Equation [7]). Given a flow rate in a pipeline, one important means of determining pressure drop is the friction factor $f,[22]$. A relationship between the friction factor, Raynolds number $R$, Pipe roughness $\epsilon$ and the pipe inner diameter $D$ is described by the Colebrook-White equation given as:

$$
\sqrt{\frac{1}{f}}=-2 \log _{10}\left(\frac{\epsilon / D}{3.7}+\frac{2.51}{R \sqrt{f}}\right)
$$

where $R>4000$. The expression in (4.33) can be written in the form of (1.1) as:

$$
f\left(f^{*}\right)=\sqrt{\frac{1}{f^{*}}}+2 \log _{10}\left(\frac{\epsilon / D}{3.7}+\frac{2.51}{R \sqrt{f^{*}}}\right),
$$

where $\epsilon / D=10^{-4}$ and $R=10^{5}$. Table 4 shows the computation results when the developed methods $\left(M_{2 i}, i=1,2,3,4,5\right)$ and the compared methods (SM and CM) are applied to solve the problem in (4.34) with an initial guess $x_{0}=0.01$. All the methods located the solution $f^{*}=$ $0.73164090877 \cdots$ of $(4.34)$ with few number of iterations and similar accuracy.

TABLE 4. Comparison of methods results for Application 1.

\begin{tabular}{ccccc}
\hline Methods & $x_{0}$ & iter & $\left|f\left(x_{k+1}\right)\right|$ & $\rho_{\text {coc }}$ \\
\hline SM & & 6 & $4.7897 \mathrm{e}-296$ & 4.0000 \\
CM & 4 & $9.0271 \mathrm{e}-109$ & 4.0370 \\
$M_{1}$ & & 5 & $3.5381 \mathrm{e}-355$ & 3.9888 \\
$M_{2}$ & & 6 & $3.7252 \mathrm{e}-210$ & 3.9623 \\
$M_{4}$ & 0.01 & 6 & $4.1820 \mathrm{e}-106$ & 3.9259 \\
$M_{6}$ & & 6 & $2.4273 \mathrm{e}-344$ & 4.0000 \\
$M_{8}$ & & 6 & $4.4259 \mathrm{e}-279$ & 3.9857 \\
$M_{10}$ & 6 & $3.03401 \mathrm{e}-192$ & 4.0000 \\
\hline
\end{tabular}

Application 2: (Population growth [2]) The expression governing the population growth is given as

$$
\frac{d N(t)}{d t}=\mu N(t)+\sigma
$$

where $N(t)$ is population at time $t, \mu$ is constant rate of birth and $\sigma$ is constant rate of immigration. The solution of the differential equation can be obtained as:

$$
N(t)=N_{0} e^{\mu t}+\frac{\sigma}{\mu}\left(e^{\mu t}-1\right)
$$

where $N_{0}$ is population at initial point ( $\mathrm{t}=0$ ). Suppose the population of certain town is 1000000 at initial point, and 43.5 percent of its population immigrate into the town in the first year, and that at the end of the year 58.6 percent of the initial population were added to the town population. It may be of interest to determine the birth rate of the town population, then we must determine $\mu$. Substitute the data in (4.36) yield

$$
f(\mu)=1586000-\frac{435000}{\mu}\left(e^{\mu}-1\right)-1000000 e^{\mu}=0 .
$$


The computation results obtained by applying the developed methods and the compared methods are presented in Table 5.

TABLE 5. Comparison of methods results for Application 2.

\begin{tabular}{ccccc}
\hline Methods & $x_{0}$ & iter & $\left|f\left(x_{k+1}\right)\right|$ & $\rho_{\text {coc }}$ \\
\hline SM & 5 & $4.9598 \mathrm{e}-265$ & 4.2742 \\
CM & & 4 & $8.9603 \mathrm{e}-138$ & 4.6000 \\
$M_{1}$ & & 5 & $1.1961 \mathrm{e}-234$ & 4.3333 \\
$M_{2}$ & & 5 & $2.1856 \mathrm{e}-303$ & 4.2676 \\
$M_{4}$ & 1 & 5 & $5.4625 \mathrm{e}-323$ & 4.2500 \\
$M_{6}$ & & 5 & $1.1112 \mathrm{e}-156$ & 4.4571 \\
$M_{8}$ & & 6 & $2.4989 \mathrm{e}-125$ & 4.6296 \\
$M_{10}$ & 5 & $9.1122 \mathrm{e}-316$ & 4.2133 \\
\hline
\end{tabular}

Application 3: (Radiation [12,20]) Consider the Plank's radiation law which calculate the energy density within an isothermal blackbody given as

$$
\varphi(\lambda)=\frac{8 \pi c h \lambda^{-5}}{e^{c h / \lambda k T}-1}
$$

where $\lambda, t, h$ and $c$ represents the radiation wavelength, blackbody absolute temperature, Boltzmann's constant, Planck's constant and $c$ speed of light. A typical radiation problem might involve calculating the wavelenth $\lambda$ which agree with maximum energy density $\varphi(\lambda)$. Differentiating Equation (4.38) with respect to $\lambda$, yield

$$
\varphi^{\prime}(\lambda)=\frac{8 \pi c h \lambda^{-6}}{e^{c h / \lambda k T}-1}\left(\frac{\left(\frac{c h}{\lambda k T}\right)}{e^{c h / \lambda k T}-1}-5\right) .
$$

From Equation (4.39), the function $\varphi(\lambda)$ attain its maxima when

$$
\frac{\left(\frac{c h}{\lambda k T}\right)}{e^{c h / \lambda k T}-1}-5=0
$$

Now, set $x=\frac{c h}{\lambda k T}$ in Equation (4.40), we get

$$
x=5-5 e^{-x}
$$

The expression in (4.41) can be written in the form of (1.1). Hence,

$$
f(x)=5-5 e^{-x}-x=0 .
$$

Obviously, $x=0$ is a trivial solution. It was discussed in [12] that a solutions of (4.42) occur near $x=5$. Here, we approximate the solution $x^{*}=4.965114231744276303698$ of (4.10) with an intial approximation $x=5$ using the methods developed here in and compared with results of some methods in Table 6. Consequently, the energy density is maximum when the approximated wavelength of radiation is $\lambda=\frac{c h}{4.965114231744276303698(k T)}$. 
TABLE 6. Comparison of methods results for Application 3.

\begin{tabular}{ccccc}
\hline Methods & $x_{0}$ & iter & $\left|f\left(x_{k+1}\right)\right|$ & $\rho_{\text {coc }}$ \\
\hline SM & & 4 & $6.9245 \mathrm{e}-374$ & 3.9787 \\
CM & & 3 & $9.3221 \mathrm{e}-169$ & 4.0238 \\
$M_{1}$ & & 3 & $2.8092 \mathrm{e}-164$ & 4.0000 \\
$M_{2}$ & & 4 & $6.9180 \mathrm{e}-374$ & 3.9787 \\
$M_{4}$ & 5 & 4 & $1.0551 \mathrm{e}-322$ & 3.9753 \\
$M_{6}$ & & 4 & $6.4843 \mathrm{e}-359$ & 3.9889 \\
$M_{8}$ & & 4 & $2.5287 \mathrm{e}-333$ & 3.9643 \\
$M_{10}$ & 4 & $6.9168 \mathrm{e}-374$ & 3.9787 \\
\hline
\end{tabular}

\section{CONCLUSiOnS}

In this paper, the method of Weerankoon and Fernando [25] have been modified with the main objective of scaling its order of convergence from three to four. This was made possible by replacing the arithmetic mean of $f^{\prime}\left(x_{k}\right)$ and $f^{\prime}\left(w_{k}\right)$ used in its second step with other means expressions and two attached weight functions $H(s)$ and $G(v)$. The analysis of convergence of the developed method reveals the conditions for which many other $\mathrm{CO}$ four methods can be constructed from it. In fact, a recent developed family of CO four methods in Lofti (2014) [13] and Chicharro et al., (2019a) [4] is one of its consequential member. The developed methods, were applied to approximate the solution of some nonlinear problems and real life problems expressed in nonlinear equation that recently appeared in literature. In all the problems solved, the computation experience from the developed methods shows promising competence in solving the problems as compared with the results of some contemporary methods. It may be of interest to extended the methods put forward in this manuscript to determining multiple roots of (1.1). Also the methods can be generalized in Banach spaces.

\section{ACKNOWLEDGMEnTS}

The authors are sincerely grateful to the Editor and anonymous referees for carefully reading the earlier versions of this manuscript and made remarks that led to its improvement.

Funding: This work enjoyed the support of the Tertiary Education Trust Fund (TETFund), Nigeria, through the Institutional Based Research (IBR) Fund.

Authors Contribution: The concept, methodology, analysis, writing and corrections are contributions of the first author in this work, while numerical implementation was done by the second author.

Interest Declaration: The authors declares no conflict of interest of any form regarding the writing and publication of this manuscript.

\section{REFERENCES}

[1] R, Behl, C. Chun, A. S. Alshormani, and S. S. Motsa, A General Way to Construct a New Optimal Scheme with Eighth-Order Convergence for Nonlinear Equations, International J. of Computational Methods, 17, No.1 (2020) 1-15.

[2] R. L. Burden, and J. D. Faires, Numerical Analysis, Brooks/Cole Publishing Company, California, 2005.

[3] P. B. Chand, F. I. Chicharro and P. Jain, On the design and analysis of high order Weerakoon-Fernando method based on weight functions, Computational and Mathematical Methods, 2, No.5 (2020) doi: $10.1002 / \mathrm{cmm} 4.1114$. 
[4] F. I. Chicharro, A. Cordero, T. H. Martinez and J. R. Torregrossa, CMMSE-2019 maen-based iterative methods for solving nonlinear chemistry problems, Journal of Mathematical Chemistry, (2019a).https://doi:10.1007/s10910-019-01085-2

[5] F. I. Chicharro, A. Cordero, N. Garrido and J. R. Torregrossa, Wide stability in a new family of optimal fourthorder iterative methods,Comp. and Math Methods, (2019b)1: Wiley https://doi.org/10.1002/cmm4.1023

[6] C. Chun, M. Y. Lee, B. Neta, and J. Dzunic, On optimal fourth-order iterative methods free from second derivative an their dynamics, Appl. Math. Comput.218,No. 11 (2012) 6427-6438.

[7] C. F. Colebrook, and C. M. White, Experiments with fluid friction in roughened pipes, Proc. R. Soc. Lond., 161 (1937) 367-381.

[8] M. Grau-Sanchez, M. Noguera, A. Grau and J. R. Herrero, A study on new computational local orders of convergence, (2012) arXiv:1202.4236[math.NA].

[9] D. Herceg, D. Herceg. Third-order modifications of Newtons method based on Stolarsky and Gini means, J. of Computational and Appl. Mathematics, 245 (2013) 53-61.

[10] D. Herceg, D. Herceg, Means based modifications of Newtons method for solving nonlinear equations, J. of Computational and Appl. Mathematics, 219 (2013) 6126-6133.

[11] D. Herceg, D. Herceg, Sixth-order modifications of Newtons method based on Stolarsky and Gini means,J. of Computational and Appl. Mathematics, 267 (2014) 244253.

[12] D. Jain, Families of Newton-like methods with fourth-order onvergence, Inter. J. of Computer Mathematics, 90, No.5 (2013) 1072-1082.

[13] T. Lofti, A new optimal method of fourth-order convergence for solving non-linear equations, Int. J. Industrial Mathematics, 6,(No.2 (2014) 1-4.

[14] T. Lukic and N.Ralevic, Geometric mean Newton's method for simple and multiple roots, Appl. Math. Lett. 21 (2008) 30-36.

[15] O. Ogbereyivwe and V. Ojo-Orobosa, High Order Quadrature Based Iterative Method for Approximating the Solution of Nonlinear Equations, Casp. J. of Math. Sc., 9, No.2 (2020) 243-255.

[16] O. Ogbereyivwe and V. Ojo-Orobosa, Family of optimal two-step fourth order iteration method and its extension for solving Nonlinear Equations, J. of Interdisciplinary Mathematics, 24, No.5 (2021) 1347-1365.

[17] O. Ogbereyivwe and K. O. Muka, Multistep Quadrature based methods for nonlinear system of equations with singular jacobian, J. of Applied Mathematics and Physics, 7, No.3 (2019) 702-725.

[18] A. M. Ostrowski, Solution of equations and systems of equations, Academic Press, New York, 1966.

[19] A. Ozban, Some new variants of Newton's method,Appl. Math. Lett, 17 (2004) 677-682.

[20] S. Parimala and J. Jayakumar, Some new higher order weighted Newton methods for solving nonlinear equation with applications, Math. and Comput. Appl. 24 (2019) 59-69.

[21] E, Sharma, S. Pandy and M. Dwivedi, New optimal fourth order iterative method for solving nonlinear equations, Int. J. on Emerging Technol, 11, No.3 (2020) 755-758.

[22] E, Shashi, Transmission pipeline calculations and Simulations Manual, Fluid flow in pipes, (Elsevier, London), (2015) 149-234.

[23] J. F. Traub, Iterative Methods for the Solution of Equations, Prentice-Hall, New Jersey, 1964.

[24] S, Weerakoon and T. G. I. Fernando, A variant of Newton's method with third-order convergence, App. Math. Lett, 13, No. 1 (2000) 87-93.

[25] Z. Xiaojain. A class of Newton's method with third-order convergence, Appl. Math. Lett, 20 (2007) 10261030 . 